\title{
Fast Implementation of Two-Dimensional Singular Spectrum Analysis for Effective Data Classification in Hyperspectral Imaging
}

\author{
Jaime Zabalza ${ }^{1,2}$, Chunmei Qing ${ }^{1}$, Peter Yuen ${ }^{3}$, Genyun Sun ${ }^{4}$, Huimin Zhao ${ }^{5,6}$, Jinchang Ren ${ }^{2}$ \\ ${ }^{1}$ Schooll of Electronic and Information Engineering, South China University of Technology, Guangzhou, China \\ ${ }^{2}$ Department of Electronic and Electrical Engineering, University of Strathclyde, Glasgow, U.K. \\ ${ }^{3}$ Centre for Electronic Warfare, Electro-Optics, Image and Signal Processing Group, Cranfield University, Swindon, U.K. \\ ${ }^{4}$ School of Geosciences, China University of Petroleum (Huadong), Qingdao, China \\ ${ }^{5}$ School of Computer Science, Guangdong Polytechnic Normal University, Guangzhou, China \\ ${ }^{6}$ The Guangzhou Key Laboratory of Digital Content Processing and Security Technologies, Guangzhou, China
}

\begin{abstract}
Although singular spectrum analysis (SSA) has been successfully applied for data classification in hyperspectral remote sensing, it suffers from extremely high computational cost, especially for 2D-SSA. As a result, a fast implementation of 2D-SSA namely F-2D-SSA is presented in this paper, where the computational complexity has been significantly reduced with a rate up to $60 \%$. From comprehensive experiments undertaken, the effectiveness of F-2D-SSA is validated producing a similar high-level of accuracy in pixel classification using support vector machine (SVM) classifier, yet with a much reduced complexity in comparison to conventional 2D-SSA.
\end{abstract} Therefore, the introduction and evaluation of F-2D-SSA completes a series of studies focused on SSA, where in this particular research, the reduction in computational complexity leads to potential applications in mobile and embedded devices such as airborne or satellite platforms.

Index Terms-Data classification, fast 2-D singular spectrum analysis (F-2D-SSA), hyperspectral imaging (HSI), land cover analysis, remote sensing.

\section{INTRODUCTION}

Data classification and recognition has become essential in many different scientific and engineering disciplines. After data acquisition and conditioning, extracting appropriate features from the data is vital for an adequate performance in the classifier stage, leading to a discriminative characterization and therefore improved classification accuracy. The introduction of hyperspectral imaging (HSI) technology in the last decades has become of great importance for several applications as it contains large amounts of data which seem especially suitable for this feature extraction, where hyperspectral images are obtained in a 3-D hyperspectral cube,

Corresponding authors: Dr C. Qing (qchm@scut.edu.cn), Dr G. Sun (genyunsun@163.com), Prof. H Zhao (zhaohuimin@gpnu.edu.cn) and Dr J Ren (jinchang.ren@strath.ac.uk). 
presenting 2-D scenes in a wide spectral range with contiguous wavelengths. This cube provides 1-D spectral signatures in each pixel, so elements in the 2-D scene can be recognized and labeled with promising accuracy in quite diverse applications such as food quality analysis [1, 2], health/medical studies [3], arts [4], or remote sensing [5, 6].

In some of our previous work, we have evaluated the singular spectrum analysis (SSA) [7] technique for feature extraction in HSI remote sensing. Basically, the SSA algorithm is able to decompose original 1-D signals into the main trend, oscillations and noise; therefore, initially we applied this technique for feature extraction in the spectral domain (applied to pixels) as 1D-SSA [8], which led to improved support vector machine (SVM) classification accuracy. Afterwards, we naturally extended this approach to the 2-D spatial domain (applied to spectral bands or images), introducing the 2D-SSA method plus a comprehensive benchmarking with the current state of the art in [9], where impressive results are achieved.

Therefore, 2D-SSA is proven to beat in terms of classification accuracy state-of-the-art techniques in HSI, including from classic methods such as the principal component analysis (PCA) [10], median filtering and morphological operators, to more modern approaches such as the 2-D empirical mode decomposition (2D-EMD) [11], the main competitor of 2D-SSA [9]. In fact, while 2D-EMD is based on empirical iterations, becoming computationally expensive, 2D-SSA is faster, being based on the well-known singular value decomposition (SVD). Other highly accurate methods such as the adaptive filter with derivative (AFD) [12] and the extended morphological profile (EMP) [13], based on median filtering and morphology respectively, are not able to achieve the accuracy provided by 2D-SSA. Overall, the potential provided by 2D-SSA is great and explains the interest and attention paid to it.

However, general SSA in HSI remote sensing requires to be applied either to every pixel (1-D case) or every spectral band (2-D case). Indeed, the individual application uses the same configuration values for every item (pixel or band), an initial simplification stated in $[8,9]$ that, additionally, leads to potential benefits. This pixel- or band-based implementation translates into reiteration of some complex steps, such as the SVD, which has inevitably resulted in substantially increased overall computational complexity. To address this issue, a fast implementation F-1D-SSA was proposed in [14], following a mathematical trick that is possible thanks to the use of the same configuration for every item (pixel). Now, it is essential to adapt and create a fast implementation for the 2-D case (F-2D-SSA) to finally complete our SSA exploration (Fig. 1). 


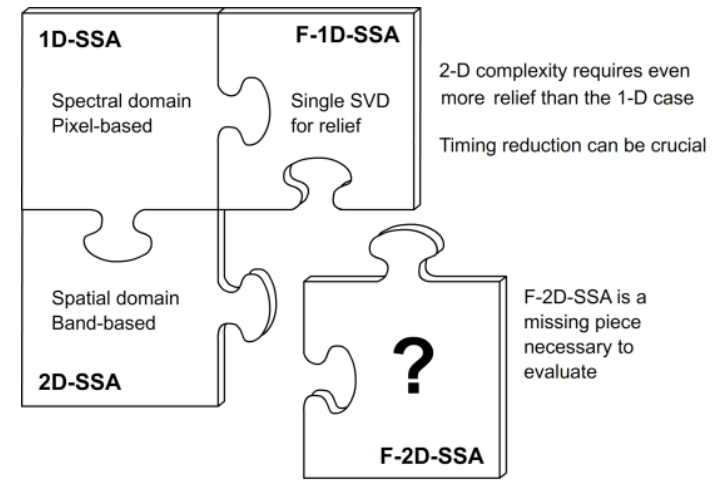

Fig. 1. F-2D-SSA inside the context of the SSA methods in hyperspectral remote sensing.

Given the potential of 2D-SSA, we find indispensable to introduce and evaluate a fast implementation of it. Actually, the 2D-SSA method, yet faster than 2D-EMD, still presents considerable computational complexity that makes crucial the introduction of computational relief and optimization. Therefore, with an increasing world-wide interest in mobile and embedded devices, accurate classification methods such as 2D-SSA are highly encouraged to optimize its implementation, reducing complexity and running time. Consequently, the main contribution presented in this paper is to propose a fast implementation for 2D-SSA, becoming a novel method, which is evaluated to show the superiority of its performance.

The remaining part of this manuscript is organized as follows. Section II starts with the basic mathematical background of the conventional 2D-SSA algorithm for feature extraction in HSI remote sensing, followed by our trick for fast implementation and the F-2D-SSA algorithm description. The experimental setup to compare both conventional and fast implementations, showing their differences under several scenarios in SVM classification, is presented in Section III. Finally, experimental results and further analysis are discussed in Section IV, leading to the concluding remarks drawn in Section V.

\section{FAST IMPLEMENTATION F-2D-SSA}

Derived from the basic SSA algorithm, the 2D-SSA method is an extension employed for 2-D signals or images [15, 16]. We already introduced and evaluated the 2D-SSA algorithm for feature extraction in HSI [9], where its conventional implementation is well-know and can be easily found in several of the cited works $[9,15,16]$. In the following, a brief summary is provided for clarity to the readers.

Let $\mathbf{P}^{2 D}$ be an image sized $N_{x} \times N_{y}$, a window $L^{2 D}$ is defined with dimensions $L^{2 D}=L_{x} \times L_{y}$, where $L_{x} \in\left[1, N_{x}\right]$ and $L_{y} \in\left[1, N_{y}\right]$. With this window, a trajectory matrix $\mathbf{X}^{2 D} \in \mathfrak{R}^{L^{2 D} \times K^{2 D}}$ of the image $\mathbf{P}^{2 D}$ can be constructed (embedding stage), where $K^{2 D}=\left(N_{x}-L_{x}+1\right)\left(N_{y}-L_{y}+1\right)$. This matrix $\mathbf{X}^{2 D}$ presents a structure called Hankel-block-Hankel (HbH) [9]. 
SVD is then applied to the matrix $\mathbf{X}^{2 D}$. This SVD is equivalent to an eigenvalue decomposition (EVD) of the matrix $\mathbf{X}^{2 D} \mathbf{X}^{2 D^{T}} \quad, \quad$ which results in eigenvalues $\quad\left(\lambda_{1} \geq \lambda_{2} \geq \cdots \geq \lambda_{L^{2 D}}\right)$ and corresponding eigenvectors $\mathbf{U}=\left(\mathbf{u}_{1}, \mathbf{u}_{2}, \cdots, \mathbf{u}_{L^{2 D}}\right) \in \mathfrak{R}^{L^{2 D} \times L^{2 D}}$. Therefore, the trajectory matrix is decomposed in $\mathbf{X}^{2 D}=\mathbf{X}_{1}+\mathbf{X}_{2}+\cdots+\mathbf{X}_{L^{2 D}}$ where each matrix $\mathbf{X}_{l}$ is related to its corresponding eigenvalue, and can be defined by

$$
\mathbf{X}_{l}=\sqrt{\lambda_{l}} \mathbf{u}_{l} \mathbf{v}_{l}^{T}, \quad \mathbf{v}_{l}=\frac{\mathbf{X}^{2 D^{T}} \mathbf{u}_{l}}{{\sqrt{\lambda_{l}}}}
$$

The 2D-SSA algorithm basically consists of decomposing an image $\mathbf{P}^{2 D}$ by SVD for a posterior reconstruction with only specific components, which is also known as grouping. In practical terms, the grouping stage consists of a multiplication derived from equations in (1), so combining both equations and selecting a single group namely $\mathbf{t}$ containing all the desired components, the reconstructed trajectory matrix is expressed as

$$
\mathbf{X}_{\mathbf{t}}^{2 D}=\mathbf{U}_{\mathbf{t}}\left(\mathbf{X}^{2 D^{T}} \mathbf{U}_{\mathbf{t}}\right)^{T}=\mathbf{U}_{\mathbf{t}} \mathbf{U}_{\mathbf{t}}^{T} \mathbf{X}^{2 D}
$$

with $\mathbf{U}_{\mathbf{t}}$ as a matrix where each column is the eigenvector from each selected component. This selection of components is known as Eigenvalue grouping (EVG). Please note that the resulting matrix $\mathbf{X}_{\mathbf{t}}^{2 D}$ from the grouping stage is not necessarily HbH type.

In order to convert this resulting matrix to the reconstructed final image, it needs to be transformed first to an HbH-type matrix. This is done by an average procedure of the different values of $\mathbf{X}_{\mathbf{t}}^{2 D}$ that contribute to the same element $(i, j)$ in the image $\mathbf{P}^{2 D}$, known as a diagonal averaging [9]. Finally, $\mathbf{Z}^{2 D}$ is the reconstructed image from the selected eigenvalue components.

Therefore, given a HSI cube, conventional implementation of the 2D-SSA method is band-based, i.e. the technique is implemented individually to every spectral image in the cube, where all spectral bands are treated equally. As shown in Fig. 2, an original scene from the cube is decomposed, where the main spatial trend and local structure is usually located in the first components. Therefore, decomposing every image in the hyperspectral cube and then selecting only the first components to reconstruct each of them individually, leads to a new cube in which the noise (usually located in the small components) is reduced. From previous explanations and our work in [9], it is clear that the performance of 2D-SSA is affected by two parameters: the window $L^{2 D}$ and the EVG grouping. How to select optimal values of configuration parameters has been previously discussed $[8,9]$, establishing noisy, lossy and intermediate regions in the 1-D case [8], where a similar interpretation is derived for the 2-D case [9]. 


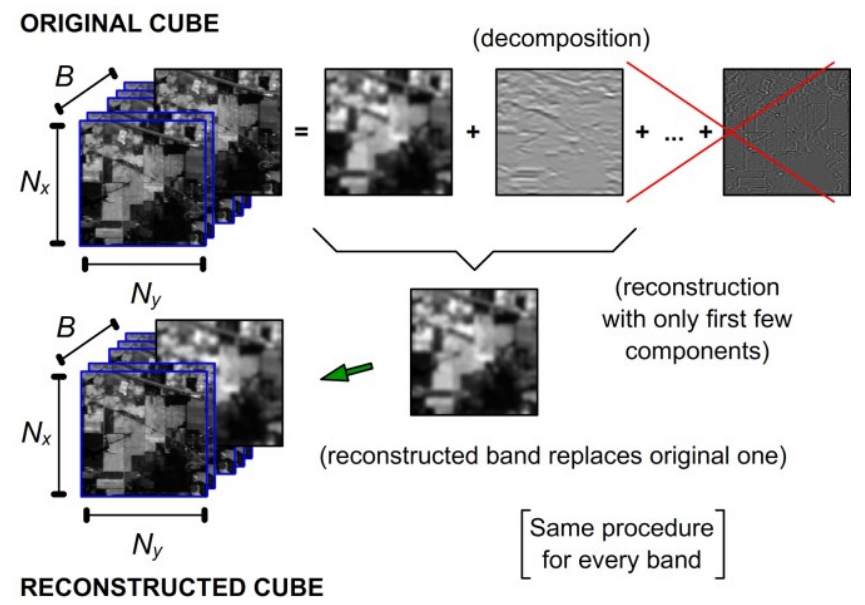

Fig. 2. Conventional application of 2D-SSA to a hyperspectral cube (band-based).

Despite the 2D-SSA method has been proven extremely effective in pixel classification, its band-based implementation forces to compute a complete SVD stage for every spectral band in the cube. This fact leads to remarkable computational complexity, which can also be reflected in large computation time. Nevertheless, given the shared configuration for each of the individual images in the cube, the $\mathbf{X}^{2 D}$ construction is undertaken in the same parameters, where the eigenvectors from the SVD can be commonly applied to all the band images. This fact allows the fast implementation trick, relieving computational complexity by implementing a unique SVD stage, as explained in the following.

The difference introduced by our fast implementation is simply related to the common SVD stage, where the rest of stages, i.e. embedding, grouping and diagonal averaging are just the same, being applied individually. Fig. 3 shows a clear comparison between conventional and fast implementation. Therefore, in our proposed F-2D-SSA, the band-based (repetitive) sequence of SVD is no longer needed. Nevertheless, a single sequence including SVD is still required.
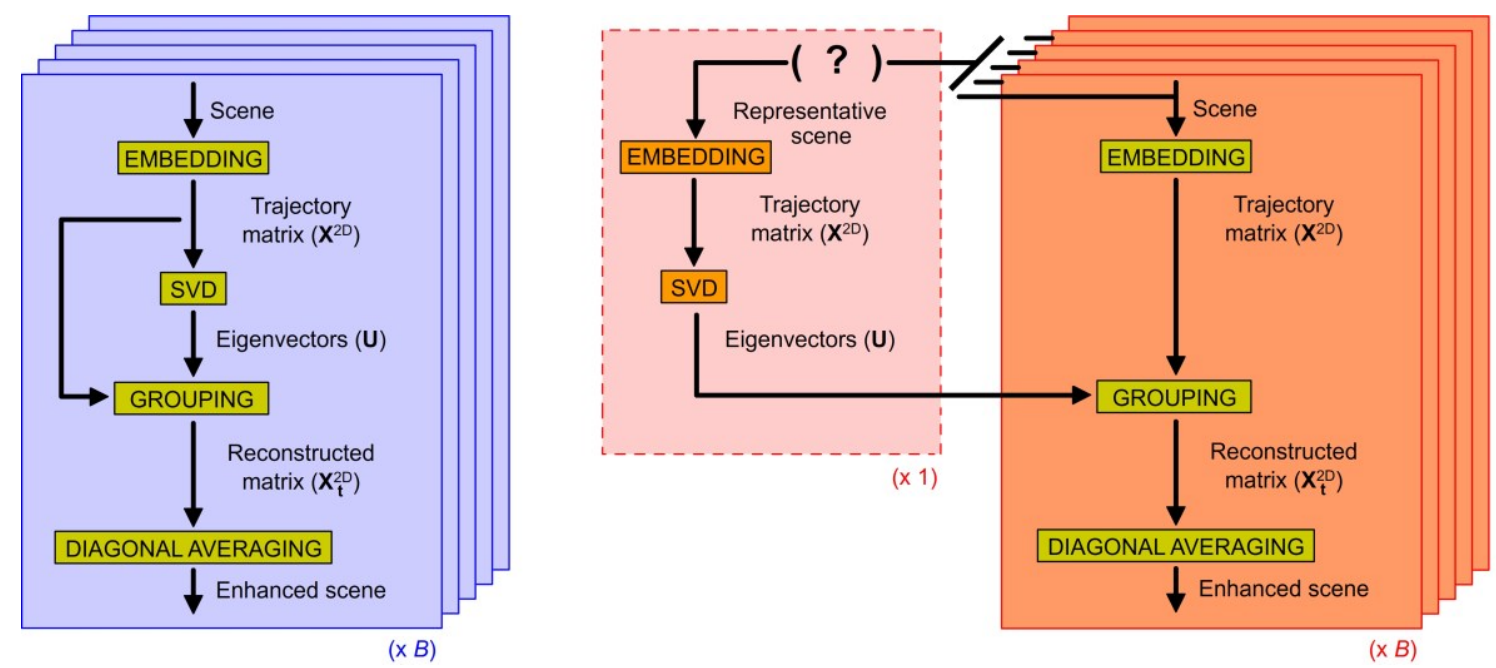

Fig. 3. Comparison of the conventional 2D-SSA (left) and the proposed F- 2D-SSA (right). 
Once the SVD stage implementation is reduced to a unique case, questions arise regarding what the appropriate SVD input is. From our point of view, the representative band scene to which the SVD is applied must possess the general characteristics of those scenes forming the hyperspectral cube. As all the scenes are indeed acquired by the same sensor, at the same time and in the same conditions, it is assumed that a scene resulting from the mean, or alternatively the median value from the scenes in the cube will contain adequately the properties of the whole data set, analogously to the 1-D case [14]. Therefore, suggesting the use of the mean (or median) image as SVD input, the implementation steps of F-2D-SSA in HSI are now listed in Algorithm 1, where experimental results are presented in Section IV to fully validate the effectiveness of the proposed F-2D-SSA algorithm.

\section{Algorithm 1: F-2D-SSA in HSI}

\section{1) Initialization:}

1.1 Input: hyperspectral cube with dimensions $N_{x} \times N_{y} \times B$;

1.2 Configuration: Choose parameters window size $L^{2 D}$, EVG and representative scene. These will be used for all the spectral scenes. It is suggested to use small/medium windows along with few eigenvalue components $\left(1^{\text {st }}, 1-2^{\text {nd }}\right)$. For the representative scene, we propose to use the mean or median scene from the whole hyperspectral cube.

\section{2) Find a unique set of eigenvalues for all the spectral bands:}

2.1 Calculate the mean or median spectral scene from the cube. It will be the representative scene;

2.2 Embed the representative scene on a trajectory matrix $\mathbf{X}^{2 D}$ using $L^{2 D}$;

2.3 Perform EVD of the matrix $\mathbf{X}^{2 D} \mathbf{X}^{2 D^{T}}$ to obtain eigenvectors $\mathbf{U}=\left(\mathbf{u}_{1}, \mathbf{u}_{2}, \cdots, \mathbf{u}_{L^{2 D}}\right) \in \mathfrak{R}^{L^{2 D} \times L^{2 D}} ;$

\section{3) Apply 2D-SSA with the given eigenvectors to one spectral band or scene $\mathbf{P}^{2 D}$ (e.g. $\left.b=1\right)$ :}

3.1 Embed the current spectral scene on a trajectory matrix $\mathbf{X}^{2 D}$ using $L^{2 D}$;

3.2 Apply eq. (2) with the unique set of eigenvectors $\mathbf{U}$ and the selected EVG ( $\mathbf{t})$ to obtain the reconstructed $\mathbf{X}_{\mathbf{t}}^{2 D}$;

3.3 Perform diagonal averaging as in [9] to invert the embedding step and obtain the final reconstructed image $\mathbf{Z}^{2 D}$.

4) Band-based repetition: Repeat the step above for the rest of spectral bands $b=2 \ldots B$. 
5) Output: A new cube with dimensions $N_{x} \times N_{y} \times B$ in which all spectral bands have been transformed by F-2D-SSA.

\section{EXPERIMENTAL SETUP}

In this paper, we propose an experimental setup similar to the one in [9], so we can compare both conventional 2D-SSA and F-2D-SSA in fair conditions to prove the advantage of the proposed fast implementation under the hardest situations. Undertaken in Matlab environment, comprehensive details about the data description and conditioning are presented below, along with the strategies for comparing methodologies and the configuration of the classifier employed (SVM).

\section{A. Data Description and Conditioning}

A total of three data sets are employed in our experiments. They are subscenes extracted from original and well-known hyperspectral images $[17,18]$ collected by two different sensors. These data sets are available to the public for remote sensing applications, and they include available ground truth allowing thus comprehensive analysis.

First, the 92AV3C data set [17] in Fig. 4 was collected by the Airborne Visible/InfraRed Imaging Spectrometer (AVIRIS) [19] in Northwest Indiana, USA. This widely-used data set contains 220 spectral bands in the range from 400 to $2500 \mathrm{~nm}$, with spatial dimensions of $145 \times 145$ pixels. However, the number of spectral bands is commonly reduced from 220 to 200 to avoid some noisy bands $[9,11,20]$. It contains 16 labeled classes related to agriculture, forest and vegetation, although it is usual to discard 7 classes with reduced number of samples available, as we do for consistency with previous studies $[8,9,11,20]$.

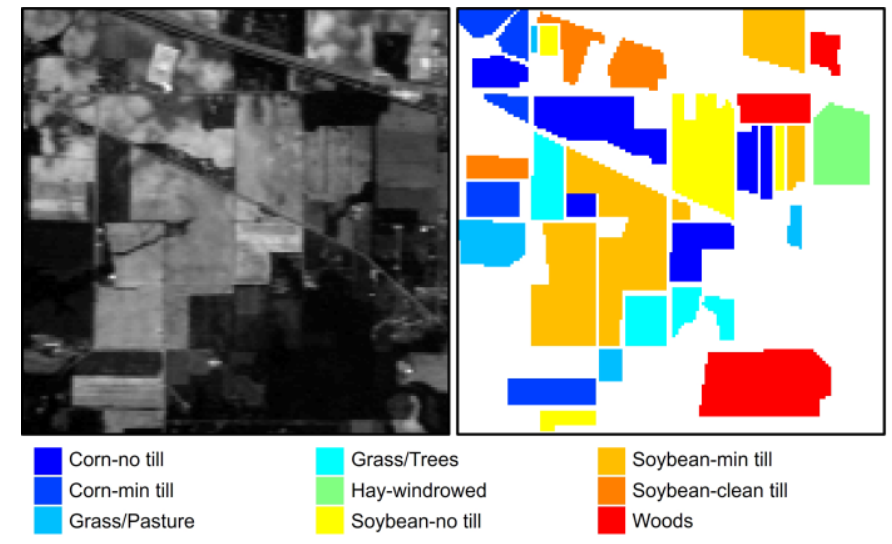

Fig. 4. One band image at the wavelength of $667 \mathrm{~nm}$ (left) and the ground truth map (right) for the 92AV3C data set. 
Second, the subscene Pavia University A (Pavia UA) taken in Pavia (North Italy) is used, where the data set was captured by the Reflective Optics System Imaging Spectrometer (ROSIS) [18, 21] and sized to $150 \times 150$ with a spatial resolution of $1.3 \mathrm{~m}$. This urban image shown in Fig. 5 presents 115 bands in the spectral range from 430 to $860 \mathrm{~nm}$, although only 103 bands are available, including 8 classes such as bitumen and asphalt among others.

Third, Salinas C image shown in Fig. 6 was acquired by AVIRIS $[18,19]$ over the Salinas Valley in California, USA. Salinas C image is sized $150 \times 150$ with 224 spectral bands and a resolution of $3.7 \mathrm{~m}$ in the spatial domain. The initial 224 spectral bands are reduced to 204, due to water absorption and noise artifact. Its ground truth provides 9 labeled classes related to agriculture such as grapes, vineyards, broccoli and fallow.

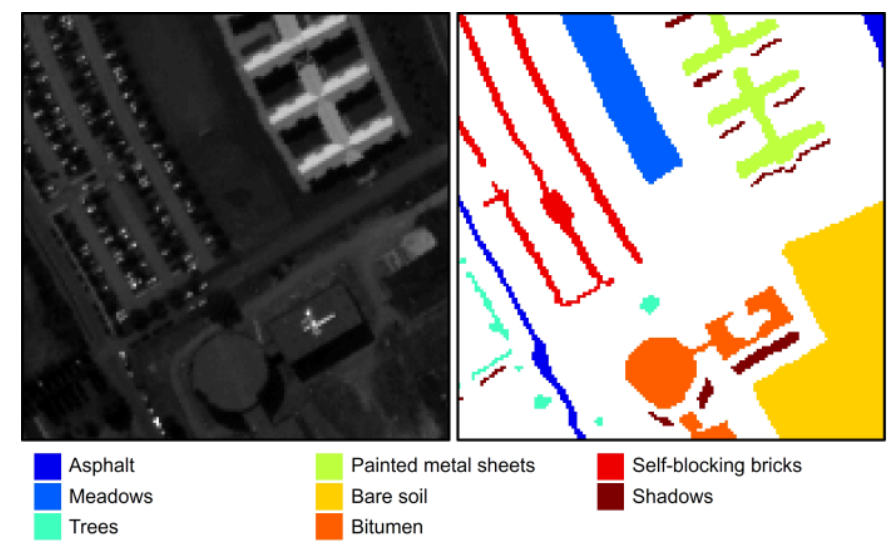

Fig. 5. One band image at the wavelength of $521 \mathrm{~nm}$ (left) and the ground truth map (right) for the Pavia UA data set.

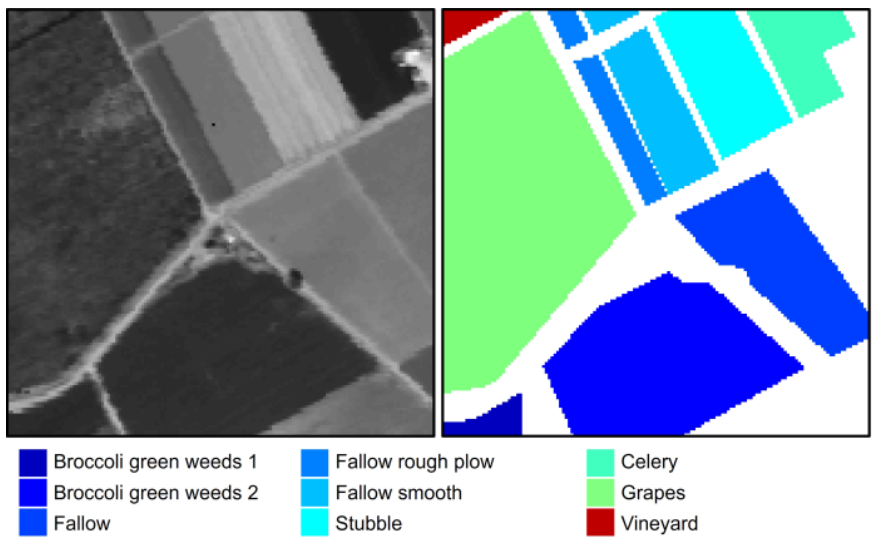

Fig.6. One band image at the wavelength of $667 \mathrm{~nm}$ (left) and the ground truth map (right) for the Salinas C data set.

\section{B. Strategies for the 2D-SSA vs F-2D-SSA Comparison}

A basic point in the experiments is to evaluate and compare the conventional 2D-SSA method with the proposed F-2D-SSA. This comparison presents two essential points; (i) to prove a similar level of classification accuracy from both methods and (ii) to show 
a decrease in computational complexity by F-2D-SSA, with reduced number of multiply-accumulates (MACs) and limited running time. In order to accomplish these two points, it is also important to evaluate the similarity of the features extracted by both methods and what an appropriate representative scene is for F-2D-SSA. Therefore, the comparison strategies developed in the experiments include similarity of extracted features, classification accuracy, analysis on the representative scene and computational complexity as detailed in Section IV.

\section{Data Classification}

Effectiveness in feature extraction is commonly measured by the accuracy achieved from the classifier in the experiments. To this end, the classification setup needs to be appropriate with relation to the current state of the art. Bearing that in mind, SVM has been proven to be very robust and adequate in multi-class classification [6, 20,22]. Additionally, the wide use of SVM in recent years has led to many and easy-to-use libraries even for embedded implementations [23, 24]. Hence, SVM is employed as classifier for supervised learning in our experiments, using the LIBSVM library available in [25] that offers a user-friendly interface with Matlab environment. For the implementation of SVM, a Gaussian RBF kernel is adopted with several works supporting this selection $[9,11$, 20], and a grid search is used every time in order to adequately tune the two key parameters from the RBF-SVM; the penalty $c$ and the gamma $\gamma$.

Every experiment using each of the feature extraction methods along with the SVM classifier is repeated ten times with different training and testing subsets (no sample overlap allowed) so that the overall experiment holds notable statistical significance. The training and testing subsets are randomly obtained through stratified sampling with an equal sample rate of $5 \%$ in each class for training, using remainder samples for testing. Then, classification results from the testing samples in terms of the overall accuracy (OA) are averaged over the ten repetitions, providing the mean values. Further evaluations also provide the mean value of the average accuracy (AA) and the class-by-class accuracy (CBC). Moreover, the McNemar's test of significance is also used as a performance measurement, where the Baseline case (use of original features) is introduced as a reference. Therefore, in our experiments McNemar's test provides a parameter $Z$ that, when $Z>1.96$, indicates the evaluated method beats the Baseline case with proper statistical significance (confidence level of 95\%). More information about McNemar's test can be found in [26].

\section{RESULTS AND EVALUATIONS}

The main purpose of the experiments is to compare our proposal F-2D-SSA with conventional 2D-SSA under the same conditions [9]. Initially, we evaluate the similarity of extracted features between both implementations to check the difference derived from the fast trick. Then, the classification accuracy achieved using different features is shown for both implementations at different configurations with parameters $L^{2 \mathrm{D}}$ and EVG. Moreover, the accuracy of both F-2D-SSA and 2D-SSA is compared with 
some state-of-the-art techniques. Afterwards, we perform a brief analysis on the representative scene to be used as a SVD input. Finally, once proven the similarity between both implementations and their superiority over the rest of techniques, their computational complexity is evaluated in terms of MACs and running time to show the clear advantage of our F-2D-SSA over the conventional case.

\section{A. Features Similarity}

The possible effects derived from the implementation of a unique SVD and the use of a single set of eigenvectors in F-2D-SSA needs to be addressed in some way. Initially, we compare an original HSI scene (a band from 92AV3C at 667nm) with the resulting scenes from conventional 2D-SSA and F-2D-SSA (for both mean and median cases). This is shown in Fig. 7, where the three resulting scenes seem unnoticeable different.

Now, the cosine distance is employed to objectively measure the difference between the proposed implementations. If the scenes being compared have similar trend, the cosine distance will detect it while other metrics such as the Euclidean distance would fail. Moreover, the cosine distance is not affected by scale and in practical terms lies in the range [0-1], making it appropriate in this context. In Table I, the mean cosine distance (comprising all spectral scenes in the cube) between the original scenes and the three 2D-SSA implementations, i.e. the conventional and the two fast implementations, is expressed for a wide range of configuration parameters. From this table, the similarity of the resulting features is clearly demonstrated.

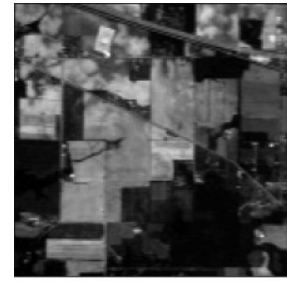

(a)

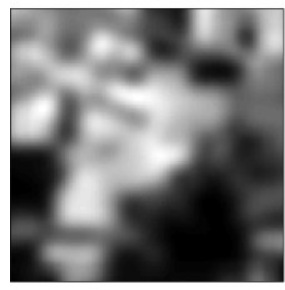

(c)

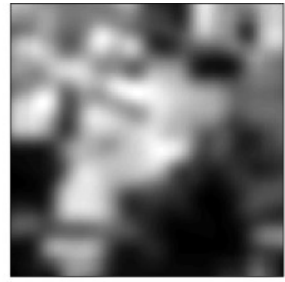

(b)

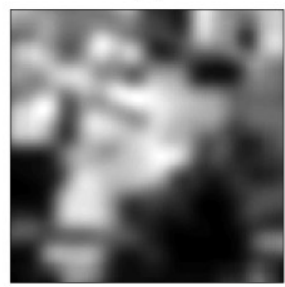

(d)

Fig. 7. Application of 2D-SSA to a scene in HSI. (a) Original scene at $667 \mathrm{~nm}$ (b) conventional 2D-SSA implementation (c) F-2D-SSA mean-based implementation (d) F-2D-SSA median-based implementation, where $L^{2 D}=10 \times 10$ and $\mathrm{EVG}=1^{\text {st }}$.

\section{TABLE I}

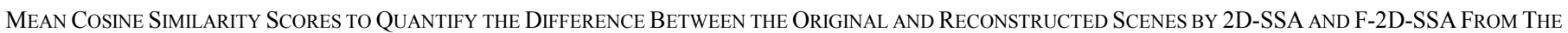
92AV3C DATA SET

\begin{tabular}{ccccc}
\hline \multicolumn{5}{c}{ Conventional SSA } \\
\hline $\boldsymbol{L}^{\mathbf{2 D}} \mid \mathbf{E V G}$ & $\mathbf{1}^{\text {st }}$ & $\mathbf{1 - 2}^{\text {nd }}$ & $\mathbf{1 - 5}^{\text {th }}$ & $\mathbf{1 - 1 0}^{\text {th }}$ \\
\hline $\mathbf{5} \times \mathbf{5}$ & 99.8996 & 99.9345 & 99.9746 & 99.9917 \\
$\mathbf{1 0} \times \mathbf{1 0}$ & 99.7999 & 99.8553 & 99.9216 & 99.9536 \\
$\mathbf{2 0} \times \mathbf{2 0}$ & 99.6737 & 99.7383 & 99.8333 & 99.8857
\end{tabular}




\begin{tabular}{ccccc}
$\mathbf{4 0} \times \mathbf{4 0}$ & 99.5288 & 99.6150 & 99.7105 & 99.7793 \\
$\mathbf{6 0} \times \mathbf{6 0}$ & 99.4519 & 99.5417 & 99.6456 & 99.7249 \\
\hline \multicolumn{5}{c}{ F-2D-SSA (mean) } \\
\hline $\boldsymbol{L}^{\mathbf{2 D}} \backslash \mathbf{E V G}$ & $\mathbf{1}^{\text {st }}$ & $\mathbf{1 - 2}^{\text {nd }}$ & $\mathbf{1 - 5}^{\text {th }}$ & $\mathbf{1 - 1 0}^{\text {th }}$ \\
\hline $\mathbf{5} \times \mathbf{5}$ & 99.8995 & 99.9343 & 99.9745 & 99.9917 \\
$\mathbf{1 0} \times \mathbf{1 0}$ & 99.7996 & 99.8547 & 99.9212 & 99.9533 \\
$\mathbf{2 0} \times \mathbf{2 0}$ & 99.6728 & 99.7374 & 99.8326 & 99.8851 \\
$\mathbf{4 0} \times \mathbf{4 0}$ & 99.5313 & 99.5874 & 99.7075 & 99.7744 \\
$\mathbf{6 0} \times \mathbf{6 0}$ & 99.4586 & 99.5045 & 99.6226 & 99.6991 \\
\hline \multicolumn{5}{c}{ F-2D-SSA (median) } \\
\hline $\boldsymbol{L}^{\mathbf{2 D}} \backslash \mathbf{E V G}$ & $\mathbf{1}^{\text {st }}$ & $\mathbf{1 - 2}$ \\
\hline $\mathbf{5} \times \mathbf{5}$ & 99.8995 & 99.9341 & 99.9742 & 99.9916 \\
$\mathbf{1 0} \times \mathbf{1 0}$ & 99.7998 & 99.8546 & 99.9208 & 99.9531 \\
$\mathbf{2 0} \times \mathbf{2 0}$ & 99.6731 & 99.7368 & 99.8324 & 99.8835 \\
$\mathbf{4 0} \times \mathbf{4 0}$ & 99.5279 & 99.6054 & 99.7062 & 99.7769 \\
$\mathbf{6 0} \times \mathbf{6 0}$ & 99.4391 & 99.5307 & 99.6359 & 99.7131 \\
\hline \hline
\end{tabular}

\section{B. Classification Accuracy Comparison}

In order to evaluate the F-2D-SSA performance, we include classification results under the same conditions for the conventional 2D-SSA and the fast implementation using both mean and median values from the whole cube as representative scenes. The results are obtained for all previous configurations of window size $L^{2 \mathrm{D}}$ and $\mathrm{EVG}(5 \times 5,10 \times 10,20 \times 20,40 \times 40$ and $60 \times 60$, with an EVG comprising the $1^{\text {st }}$, the $1-2^{\text {nd }}$, the $1-5^{\text {th }}$ and the $1-10^{\text {th }}$ components) showing the best case and the average value from all settings.

As derived from Tables II-IV, F-2D-SSA is able to provide a very similar accuracy, where mean OA values fluctuate close to the conventional ones. For instance, in the $92 \mathrm{AV} 3 \mathrm{C}$ data set, $95.66 \%$ and $95.82 \%$ are the accuracies from F-2D-SSA, compared to the conventional result of $95.71 \%$. Similar outcome is obtained from Pavia UA $(98.15 \%$ and $98.51 \%$ for $98.21 \%)$ and Salinas C (99.27\% and $99.58 \%$ for $99.81 \%)$. This consistency is also reflected by the McNemar's test parameter in brackets, having the Baseline case (original features) as reference.

TABLE II

Mean Overall ACCuracy (\%) AND Mean McNemar’s Test [Z] FOR the 92AV3C Data Set Using 2D-SSA AND F-2D-SSA

\begin{tabular}{rcc}
\hline \multirow{2}{*}{ Method } & Best case & $\begin{array}{c}\text { Average from all } \\
\text { configurations }\end{array}$ \\
\cline { 2 - 3 } & $\boldsymbol{L}^{\mathbf{2 D}}=\mathbf{1 0} \times \mathbf{1 0} \mathbf{~ E V G}=\mathbf{1}^{\text {st }}$ & $93.19[+25.9]$ \\
\hline 2D-SSA & $95.71[+31.4]$ & $93.50[+26.5]$ \\
\hline F-2D-SSA (mean) & $95.66[+31.3]$ & $93.44[+26.4]$ \\
\hline \hline F-2D-SSA (median) & $95.82[+31.5]$ & \\
\hline \hline
\end{tabular}

TABLE III

Mean Overall Accuracy (\%) ANd Mean MCNemar’s Test [Z] For the PaVia UA Data Set Using 2D-SSA AND F-2D-SSA

\begin{tabular}{|c|c|c|}
\hline Method & $\begin{array}{c}\text { Best case } \\
L^{2 D}=5 \times 5 \mathrm{EVG}=1-2^{\text {nd }}\end{array}$ & $\begin{array}{c}\text { Average from all } \\
\text { configurations }\end{array}$ \\
\hline 2D-SSA & $98.21[+8.55]$ & $96.99[+3.91]$ \\
\hline F-2D-SSA (mean) & $98.15[+8.20]$ & $96.61[+2.88]$ \\
\hline F-2D-SSA (median) & $98.51[+9.69]$ & $96.79[+3.41]$ \\
\hline
\end{tabular}

TABLE IV

Mean Overall Accuracy (\%) AND Mean McNemar’s Test [Z] For the Salinas C Data Set Using 2D-SSA AND F-2D-SSA 


\begin{tabular}{rcc}
\hline \hline \multirow{2}{*}{ Method } & Best case & $\begin{array}{c}\text { Average from all } \\
\text { configurations }\end{array}$ \\
\cline { 2 - 3 } $\boldsymbol{L}^{\mathbf{2 D}}=\mathbf{4 0} \times \mathbf{4 0} \mathbf{~ E V G}=\mathbf{1 - 2}^{\mathbf{n}}$ & $99.81[+13.6]$ & $99.44[+10.1]$ \\
\hline 2D-SSA & $99.27[+8.12]$ & $99.40[+9.72]$ \\
\hline F-2D-SSA (mean) & $99.27[+11.3]$ & $99.38[+9.47]$ \\
\hline F-2D-SSA (median) & $99.58[+1.3]$
\end{tabular}

Additionally, $\mathrm{CBC}$ and AA values (from the best case) are provided in Tables V-VII to further compare the methods accuracy, where it can be checked in detail through the different land-cover classes.

TABLE V

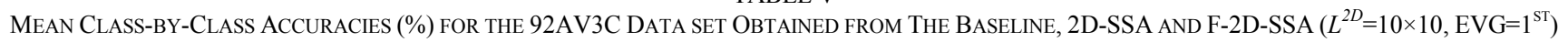

\begin{tabular}{cccccc}
\hline \multicolumn{6}{c}{ APPROACHES AS WELL AS THE NUMBER OF SAMPLES (NOS) IN EACH CLASS } \\
\hline Class & NoS & Baseline & 2D-SSA & $\begin{array}{c}\text { F-2D-SSA } \\
\text { (mean) }\end{array}$ & $\begin{array}{c}\text { F-2D-SSA } \\
\text { (median) }\end{array}$ \\
\hline$\square$ & 1434 & 75.38 & 95.38 & 94.88 & 95.65 \\
834 & 63.32 & 96.00 & 96.00 & 96.00 \\
497 & 89.30 & 94.79 & 94.77 & 94.60 \\
747 & 96.81 & 96.59 & 96.47 & 96.50 \\
489 & 99.07 & 97.09 & 97.16 & 97.11 \\
968 & 65.97 & 90.45 & 89.76 & 91.23 \\
2468 & 81.10 & 96.54 & 96.92 & 96.60 \\
$\square 14$ & 69.97 & 93.86 & 93.84 & 93.67 \\
$\square$ & 1294 & 97.62 & 98.47 & 98.45 & 98.46 \\
\hline$\square$ & Average accuracy & 82.06 & 95.46 & 95.36 & 95.54 \\
Overall accuracy & 81.26 & 95.71 & 95.66 & 95.82 \\
\hline
\end{tabular}

TABLE VI

Mean Class-By-Class Accuracies (\%) For the Pavia UA Data Set ObTained From The Baseline, 2D-SSA AND F-2D-SSA $\left(L^{2 D}=5 \times 5\right.$, EVG=1-2 $\left.{ }^{\mathrm{ND}}\right)$ APPROACHES AS WELL AS THE NUMBER OF SAMPLES (NOS) IN EACH ClasS

\begin{tabular}{cccccc}
\hline \hline Class & NoS & Baseline & 2D-SSA & $\begin{array}{c}\text { F-2D-SSA } \\
\text { (mean) }\end{array}$ & $\begin{array}{c}\text { F-2D-SSA } \\
\text { (median) }\end{array}$ \\
\hline$\square$ & 310 & 80.71 & 94.15 & 92.04 & 94.29 \\
& 957 & 97.03 & 99.90 & 99.89 & 99.78 \\
154 & 93.97 & 93.56 & 90.41 & 90.68 \\
698 & 99.40 & 99.20 & 99.61 & 99.61 \\
2559 & 96.76 & 98.98 & 99.53 & 99.55 \\
860 & 93.15 & 95.21 & 94.98 & 96.56 \\
$\square$ & 854 & 95.86 & 98.19 & 97.37 & 97.78 \\
$\square$ & 293 & 100 & 99.21 & 99.03 & 99.21 \\
\hline Average accuracy & 94.61 & 97.30 & 96.61 & 97.18 \\
Overall accuracy & 95.83 & 98.21 & 98.15 & 98.51 \\
\hline \hline
\end{tabular}

TABLE VII

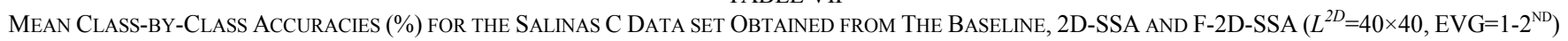
APPROACHES AS WELL AS THE NUMBER OF SAMPLES (NOS) IN EACH CLASS

\begin{tabular}{cccccc}
\hline \hline Class & NoS & Baseline & 2D-SSA & $\begin{array}{c}\text { F-2D-SSA } \\
\text { (mean) }\end{array}$ & $\begin{array}{c}\text { F-2D-SSA } \\
\text { (median) }\end{array}$ \\
\hline$\square$ & 240 & 90.53 & 99.96 & 92.15 & 95.44 \\
$\square$ & 3400 & 99.81 & 99.99 & 99.66 & 99.83
\end{tabular}




\begin{tabular}{ccccc}
1957 & 99.28 & 99.99 & 99.92 & 99.86 \\
599 & 99.16 & 98.42 & 96.73 & 98.15 \\
1155 & 96.92 & 99.29 & 97.96 & 99.04 \\
1414 & 99.96 & 99.93 & 98.70 & 99.82 \\
848 & 99.60 & 99.76 & 99.70 & 99.70 \\
5890 & 99.47 & 99.90 & 99.89 & 99.87 \\
159 & 07.28 & 98.61 & 92.98 & 92.52 \\
\hline$\square$ Average accuracy & 88.00 & 99.54 & 97.52 & 98.25 \\
Overall accuracy & 98.30 & 99.81 & 99.27 & 99.58 \\
\hline \hline
\end{tabular}

Given the classification accuracy achieved by the F-2D-SSA, now we place this performance in context with other state-of-the-art techniques. These techniques include not only using the original features (Baseline case), but also the 1D-SSA [8], the AFD [12], and the 2D-EMD [11] approaches, where in all of them the original dimensionality of features is preserved, so no data reduction is achieved. On the other hand, the EMP method [13] along with the combination of either 2D-SSA or F-2D-SSA with PCA [10] is evaluated for both feature extraction and data reduction. All the methods are implemented with the optimized parameters used in [9] showing the best result.

Our proposal presents similar results as the conventional 2D-SSA; hence, it is proven to beat most of the techniques as shown in Table VIII, where only few cases provide higher accuracy (AFD, 2D-EMD and EMP for Pavia UA). Results from Table VIII are the best cases obtained in every method [9]. Therefore, 2D-SSA and F-2D-SSA are configured with $L^{2 D}=10 \times 10, E V G=1^{\mathrm{st}}, L^{2 D}=5 \times 5$, $\mathrm{EVG}=1-2^{\text {nd }}$ and $L^{2 D}=40 \times 40, \mathrm{EVG}=1-2^{\text {nd }}$ for $92 \mathrm{AV} 3 \mathrm{C}$, Pavia UA and Salinas C, respectively. Moreover, our fast implementation can also be combined with the PCA technique for data reduction. This combination was already evaluated in [9], so both the 2D-SSA-PCA previously, and now F-2D-SSA-PCA are able to exploit not only the spatial but also the spectral domain of HSI cubes. This combination achieves the best results from all the techniques evaluated, even though the number of features is reduced.

TABLE VIII Mean Overall Accuracy (\%) and Mean McNemar’s Test [Z] From the DifFerent Methods Evaluated (Best Cases)

\begin{tabular}{cccc}
\hline \hline Method & 92AV3C & Pavia UA & Salinas C \\
\hline \multicolumn{4}{c}{ ORIGINAL DIMENSION OF FEATURES } \\
\hline Baseline & $81.26[-0.00]$ & $95.83[-0.00]$ & $98.30[-0.00]$ \\
\hline 1D-SSA [8] & $85.50[+11.4]$ & $95.53[-1.88]$ & $98.52[+3.41]$ \\
\hline AFD [12] & $95.11[+30.9]$ & $99.32[+13.0]$ & $99.70[+12.8]$ \\
\hline 2D-EMD [11] & $95.28[+31.7]$ & $99.53[+14.6]$ & $99.71[+13.8]$ \\
\hline 2D-SSA [9] & $95.71[+31.4]$ & $98.21[+8.55]$ & $99.81[+13.6]$ \\
\hline F-2D-SSA & $95.82[+31.5]$ & $98.51[+9.69]$ & $99.58[+11.3]$ \\
\hline \multicolumn{4}{c}{ DATA REDUCTION (dimension of features) } \\
\hline EMP [13] & $94.83[+29.3]$ & $99.56[+14.1]$ & $99.49[+10.5]$ \\
2D-SSA-PCA & $97.61[+35.5]$ & $99.58[+14.1]$ & $99.83[+14.0]$ \\
[9] & $(15)$ & $(20)$ & $(20)$ \\
\hline F-2D-SSA-PCA & $\mathbf{9 7 . 5 9}[+35.5]$ & $\mathbf{9 9 . 5 9}[+\mathbf{1 4 . 2}]$ & $\mathbf{9 9 . 8 8 ~ [ + 1 4 . 5 ]}$ \\
& $(\mathbf{1 5 )}$ & $(\mathbf{2 0})$ & $(\mathbf{1 5 )}$ \\
\hline \hline
\end{tabular}


C. Analysis on the Representative Scene

Although the mean and median scenes from the whole HSI cube seem an appropriate input for F-2D-SSA, it is important to remark that other different options are possible. In order to bring some light to this issue, we briefly evaluate the performance of different inputs, in terms of OA. In fact, now we use every single spectral band in the cube as a representative scene to see how the classification accuracy does with relation to the new input.

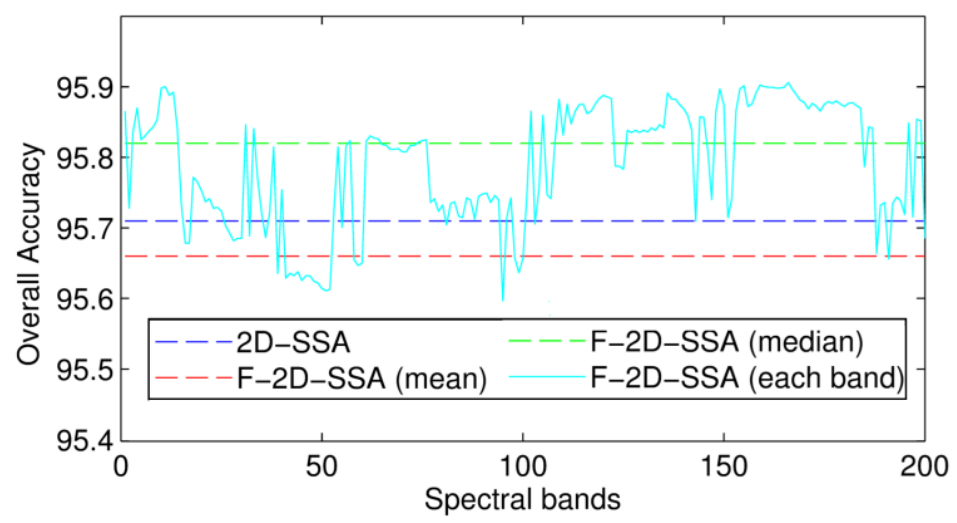

Fig.8. Comparison of the OA for F-2D-SSA $\left(L^{2 D}=10 \times 10, E V G=1^{\text {st }}\right)$ with each spectral band used as a representative scene for the $92 \mathrm{AV} 3 \mathrm{C}$ data set.

In Fig. 8, the OA values obtained with the best configuration for the 92AV3C data set fluctuate for the different spectral bands used as inputs. As can be seen, most of the values are found between the F-2D-SSA (mean) and the F-2D-SSA (median) cases, which actually validates the use of the mean and median operators for obtaining a representative scene. On the other hand, it is also observed that the use of some specific bands can slightly increase $(b=160-170)$ or degrade $(b=40-50)$ the performance, yet all OA values are close to the conventional 2D-SSA case. A similar behavior is found for the other data sets (Fig. 9-10).

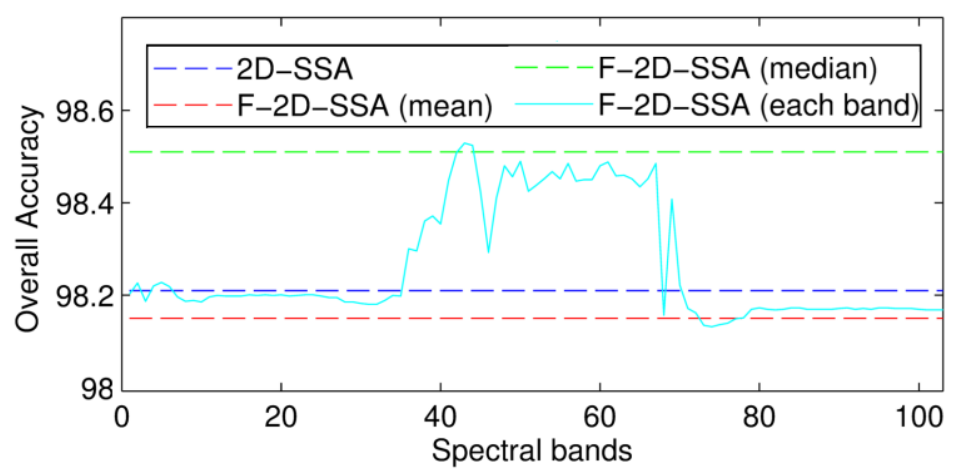

Fig.9. Comparison of OA of classification for F-2D-SSA $\left(L^{2 D}=5 \times 5, E V G=1-2^{\text {nd }}\right)$ using each spectral band as a representative scene for Pavia UA. 


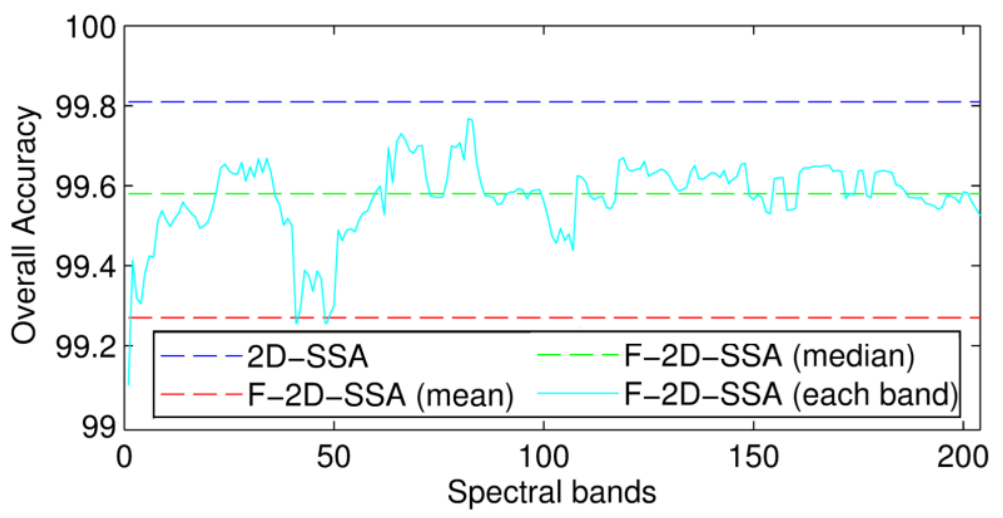

Fig.10. Comparison of the OA of classification for F-2D-SSA $\left(L^{2 D}=40 \times 40, E V G=1-2^{\text {nd }}\right)$ using each spectral band as a representative scene for Salinas $C$.

From the behavior detected in Figs. 8-10, the median scene from the cubes seems to perform better than the mean scene. The explanation to this fact is simple; the median operator, unlike others such as the mean (average), is not mainly influenced by outliers, becoming more robust. In other words, given that HSI cubes usually contain noisy bands, the representative scene must avoid as much as possible noisy content, and the median operator smartly disregards noisy outliers. Actually, the median performs better than the majority of individual bands. Overall, we can suggest as a general recommendation the use of the median scene as input to the unique SVD in the fast implementations.

\section{Computational Complexity}

The proposed fast implementation reduces the SVD computation from hundreds of times to a single case that is applied to a representative scene. This fact directly translates into a saving factor in MACs related to the SVD step, as shown in this subsection, where we briefly analyze the computational complexity derived from each stage as follows:

- In the embedding procedure, an original image is relocated into a trajectory matrix by means of a window with size $L^{2 \mathrm{D}}$, however, no MACs are involved in this operation.

- Then, the SVD stage takes places. This SVD can actually be computed by several algorithms described in the literature, where equivalent implementations based on EVD are also possible. As stated in [14], an equivalent EVD applied to $\mathbf{S}=\mathbf{X}^{2 D}\left(\mathbf{X}^{2 D}\right)^{T}$ is simpler $\left(\left(L^{2 D}\right)^{2} K^{2 D}+\left(L^{2 D}\right)^{3}\right)$ than the SVD complexity $\left(\left(L^{2 D}\right)^{2} K^{2 D}+L^{2 D}\left(K^{2 D}\right)^{2}+\left(K^{2 D}\right)^{3}\right)$ suggested in $[27,28]$, therefore, we work with this EVD complexity.

- Afterwards, selection of components is made. The grouping stage is represented by the equation in (2), and even though in [14] we computed the multiplication $\mathbf{U}_{\mathbf{t}} \mathbf{U}_{\mathbf{t}}^{T}$, actually computing the two multiplications from $\mathbf{U}_{\mathbf{t}}\left(\mathbf{U}_{\mathbf{t}}^{T} \mathbf{X}^{2 D}\right)$ keeping the order from brackets is less complex, so the complexity is stated as $\left(2 L^{2 D} K^{2 D} p\right)$ instead of $\left(\left(L^{2 D}\right)^{2} p+\left(L^{2 D}\right)^{2} K^{2 D}\right)$. 
- Finally, the last stage corresponding to the diagonal averaging procedure can be approximated as $N_{\mathrm{x}} N_{y}$ in a similar way as we did in [14].

In Table IX, the computational complexity comparison between both implementations can be seen for all stages, where following Tables X-XII provide the actual number of MACs and the saving factor achieved for the three data sets, given four varied configurations. The complexity reduction in the SVD stage makes the fast implementation clearly easier than the conventional one, with minimum saving factors of 2 (halving the number of MACs) and even achieving a dramatically high reduction superior to 100 depending on the configuration case.

TABLE IX

COMPUTATIONAL COMPLEXITY IN THE DiFFERENT STAGES

\begin{tabular}{cccc}
\hline \hline Stage & 2D-SSA & F-2D-SSA & $\begin{array}{c}\text { Saving } \\
\text { factor }\end{array}$ \\
\hline Embed. & N/A & N/A & 1 \\
\hline SVD & {$\left[\left(L^{2 D}\right)^{2} K^{2 D}+\left(L^{2 D}\right)^{3}\right] \times B$} & {$\left[\left(L^{2 D}\right)^{2} K^{2 D}+\left(L^{2 D}\right)^{3}\right] \times 1$} & $B$ \\
\hline Grouping & {$\left[2 L^{2 D} K^{2 D} p\right] \times B$} & {$\left[2 L^{2 D} K^{2 D} p\right] \times B$} & 1 \\
\hline D. Av. & {$\left[N_{\mathrm{x}} N_{y}\right] \times B$} & {$\left[N_{\mathrm{x}} N_{y}\right] \times B$} & 1 \\
\hline \hline
\end{tabular}

TABLE X

COMPUTATIONAL COMPLEXITY (MACS) AND SAVING FACTOR FOR THE 92AV3C DATA SET IN DiFFERENT CONFIGURATIONS

\begin{tabular}{ccccc}
\hline \hline $\begin{array}{c}\boldsymbol{L}^{\mathbf{2 D}}= \\
\mathbf{E V G}=\end{array}$ & $\begin{array}{c}\mathbf{5} \times \mathbf{5} \\
\mathbf{1}^{\text {st }}\end{array}$ & $\begin{array}{c}\mathbf{5} \times \mathbf{5} \\
\mathbf{1 - 1 0}^{\text {th }}\end{array}$ & $\begin{array}{c}\mathbf{6 0 \times 6 0} \\
\mathbf{1}^{\text {st }}\end{array}$ & $\begin{array}{c}\mathbf{6 0} \times \mathbf{6 0} \\
\mathbf{1 - 1 0}^{\text {th }}\end{array}$ \\
\hline 2D-SSA & $2691 \mathrm{e} 6$ & $4481 \mathrm{e} 6$ & $28512 \mathrm{e} 9$ & $28608 \mathrm{e} 9$ \\
\hline F-2D-SSA & $215 \mathrm{e} 6$ & $2005 \mathrm{e} 6$ & $153 \mathrm{e} 9$ & $249 \mathrm{e} 9$ \\
\hline Saving factor & 12.5 & 2.23 & 186 & 115 \\
\hline \hline
\end{tabular}

TABLE XI

COMPUtATIONAL COMPLEXITy (MACs) AND SAVING FACTOR FOR THE PAVIA UA DATA SET IN DiFFERENT CONFIGURATIONS

\begin{tabular}{ccccc}
\hline \hline $\begin{array}{c}\boldsymbol{L}^{\mathbf{2 D}}= \\
\mathbf{E V G}=\end{array}$ & $\begin{array}{c}\mathbf{5} \times \mathbf{5} \\
\mathbf{1}^{\text {st }}\end{array}$ & $\begin{array}{c}\mathbf{5} \times \mathbf{5} \\
\mathbf{1 - 1 0}^{\text {th }}\end{array}$ & $\begin{array}{c}\mathbf{6 0 \times} \times \mathbf{6 0} \\
\mathbf{1}^{\text {st }}\end{array}$ & $\begin{array}{c}\mathbf{6 0 \times 6 0} \\
\mathbf{1 - 1 0}^{\text {th }}\end{array}$ \\
\hline 2D-SSA & $1486 \mathrm{e} 6$ & $2474 \mathrm{e} 6$ & $15866 \mathrm{e} 9$ & $15921 \mathrm{e} 9$ \\
\hline F-2D-SSA & $125 \mathrm{e} 6$ & $1113 \mathrm{e} 6$ & $160 \mathrm{e} 9$ & $215 \mathrm{e} 9$ \\
\hline Saving factor & 11.8 & 2.22 & 99.1 & 73.9 \\
\hline \hline
\end{tabular}

TABLE XII

COMPUTATIONAL COMPLEXITY (MACS) AND SAVING FACTOR FOR THE SALINAS C DATA SET IN DiFFERENT CONFIGURATIONS

\begin{tabular}{ccccc}
\hline \hline $\begin{array}{c}\boldsymbol{L}^{\mathbf{2}}= \\
\mathbf{E V G}=\end{array}$ & $\begin{array}{c}\mathbf{5} \times \mathbf{5} \\
\mathbf{1}^{\text {st }}\end{array}$ & $\begin{array}{c}\mathbf{5} \times \mathbf{5} \\
\mathbf{1 - 1 0}^{\text {th }}\end{array}$ & $\begin{array}{c}\mathbf{6 0 \times 6 \mathbf { 6 0 }} \\
\mathbf{1}^{\text {st }}\end{array}$ & $\begin{array}{c}\mathbf{6 0 \times 6 0} \\
\mathbf{1 - 1 0}^{\text {th }}\end{array}$ \\
\hline 2D-SSA & $2943 \mathrm{e} 6$ & $4900 \mathrm{e} 6$ & $31424 \mathrm{e} 9$ & $31533 \mathrm{e} 9$ \\
\hline F-2D-SSA & $235 \mathrm{e} 6$ & $2192 \mathrm{e} 6$ & $166 \mathrm{e} 9$ & $276 \mathrm{e} 9$ \\
\hline Saving factor & 12.5 & 2.24 & 189 & 114 \\
\hline \hline
\end{tabular}

This reduced complexity translates into faster running times of the algorithms, as derived from Tables XIII-XV. Configuration with larger windows $L^{2 \mathrm{D}}$ (here the EVG parameter has little influence) take clear advantage of the fast implementations, where the 
total timing can be reduced up to $60 \%$, going from 496 to $194 \mathrm{~s}$ for the $92 \mathrm{AV} 3 \mathrm{C}$ data set, where the timing reduction in percentage is similar for the other data sets.

TABLE XIII

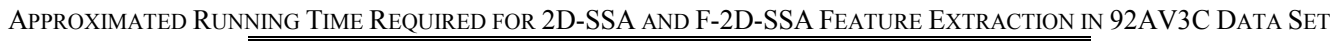

\begin{tabular}{|c|c|c|c|c|}
\hline \multirow{2}{*}{$\frac{\text { Parameters }}{L^{2 D}}$} & \multirow{2}{*}{ 2D-SSA } & \multirow{2}{*}{ F-2D-SSA } & \multicolumn{2}{|c|}{ Reduction } \\
\hline & & & (s) & (\%) \\
\hline $5 \times 5$ & 19 & 18 & 1 & 5.26 \\
\hline $10 \times 10$ & 31 & 28 & 3 & 9.68 \\
\hline $20 \times 20$ & 69 & 58 & 11 & 15.9 \\
\hline $40 \times 40$ & 244 & 137 & 107 & 43.9 \\
\hline $60 \times 60$ & 496 & 194 & 302 & 60.9 \\
\hline
\end{tabular}

TABLE XIV

ApProximated RunNing Time ReQuired FOR 2D-SSA AND F-2D-SSA FEATURE EXTRACTION IN PAVIA UA Data SET

\begin{tabular}{crrrr}
\hline \hline Parameters & \multirow{2}{*}{ 2D-SSA } & \multirow{2}{*}{ F-2D-SSA } & \multicolumn{3}{c}{ Reduction } \\
\cline { 1 - 4 } $\boldsymbol{L}^{\mathbf{2}}$ & 10 & 10 & 0 & $\mathbf{( \% )}$ \\
\hline $\mathbf{5} \times \mathbf{5}$ & 17 & 15 & 2 & 0.00 \\
$\mathbf{1 0} \times \mathbf{1 0}$ & 38 & 32 & 6 & 11.8 \\
$\mathbf{2 0} \times \mathbf{2 0}$ & 137 & 77 & 60 & 43.8 \\
$\mathbf{4 0} \times \mathbf{4 0}$ & 282 & 113 & 169 & 59.9 \\
$\mathbf{6 0} \times \mathbf{6 0}$ & & & & \\
\hline
\end{tabular}

TABLE XV

APProximated RunNing Time ReQuired For 2D-SSA AND F-2D-SSA FEATURE EXTRACTION IN SALINAS C DATA SET

\begin{tabular}{crrrr}
\hline \hline Parameters & \multirow{2}{*}{ 2D-SSA } & \multirow{2}{*}{ F-2D-SSA } & \multicolumn{2}{c}{ Reduction } \\
\cline { 1 - 3 } $\boldsymbol{L}^{\boldsymbol{2 \boldsymbol { D }}}$ & & & $\mathbf{( s )}$ & $\mathbf{( \% )}$ \\
\hline $\mathbf{5} \times \mathbf{5}$ & 20 & 19 & 1 & 5.00 \\
$\mathbf{1 0} \times \mathbf{1 0}$ & 34 & 30 & 4 & 11.8 \\
$\mathbf{2 0} \times \mathbf{2 0}$ & 75 & 64 & 11 & 14.7 \\
$\mathbf{4 0} \times \mathbf{4 0}$ & 270 & 150 & 120 & 44.4 \\
$\mathbf{6 0} \times \mathbf{6 0}$ & 557 & 222 & 335 & 60.1 \\
\hline \hline
\end{tabular}

In Fig. 11, we represent the running time in the different stages of conventional 2D-SSA for a better understanding. The growth of the SVD step when increasing the window size $L^{2 \mathrm{D}}$ results noticeable in every data set. Therefore, while the increment in the rest of stages is modest, the SVD stage dramatically rise, achieving $60 \%$ of the total timing for $L^{2 \mathrm{D}}=60 \times 60$. Again, that demonstrates the advantage of our fast implementation and its potential for portable and limited-resources applications.
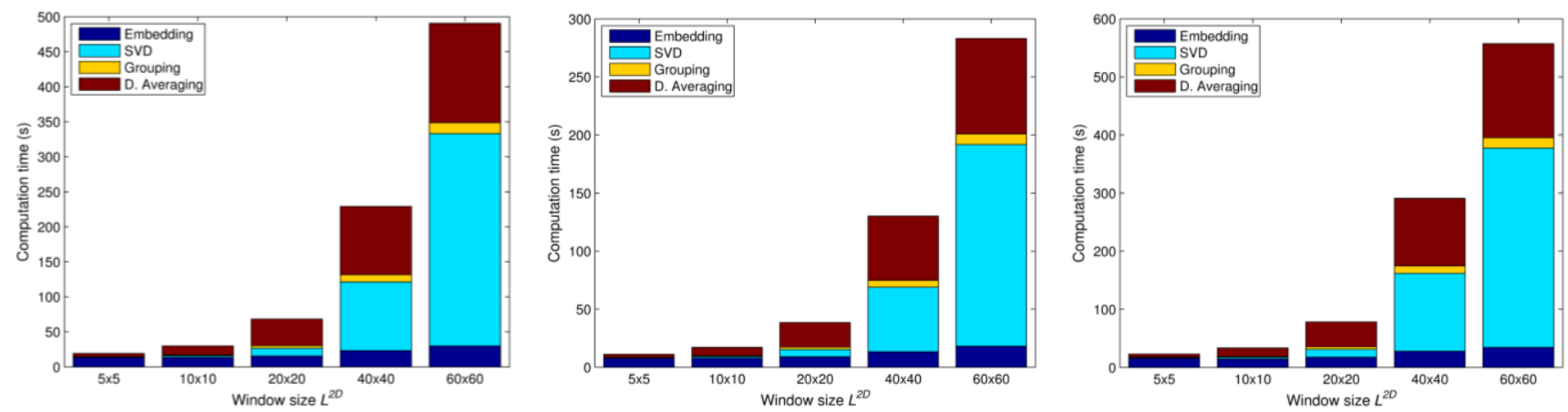
Fig.11. Running time (s) per stage and different $L^{2 D}$ in conventional 2D-SSA for 92AV3C (left), Pavia UA (middle) and Salinas C (right).

Finally, a global comparison of the SSA methodologies, with classification accuracy and running time, is provided in Table XVI. The better performance of the 2-D methodologies in classification accuracy is clear, however, the pixel-based implementation from the 1-D cases involves a faster running time, basically because trajectory matrices in 1-D are smaller. Discussions can be derived from this fact, regarding what a good trade-off between accuracy and complexity can be when working with HSI. From our point of view, the classification accuracy comes first; looking for accuracies close to $100 \%$, and that is probably the reason why most efforts in HSI are focused on the highest-accuracy problem. Nevertheless, complexity is a factor to bear in mind, making some implementations unfeasible. A good example is 2D-EMD in Salinas C, where it requires 1056 s, something incompatible with fast tasks. This issue points out our fast implementation importance and contribution.

TABLE XVI

\begin{tabular}{|c|c|c|c|c|c|c|}
\hline & \multicolumn{2}{|c|}{ 92AV3C } & \multicolumn{2}{|c|}{ Pavia UA } & \multicolumn{2}{|c|}{ Salinas C } \\
\hline Method & $\begin{array}{l}\mathbf{O A} \\
(\%)\end{array}$ & $\begin{array}{c}\text { time } \\
\text { (s) }\end{array}$ & $\begin{array}{l}\mathbf{O A} \\
(\%)\end{array}$ & $\begin{array}{c}\text { time } \\
\text { (s) }\end{array}$ & $\begin{array}{l}\mathrm{OA} \\
(\%)\end{array}$ & $\begin{array}{c}\text { time } \\
\text { (s) }\end{array}$ \\
\hline Baseline & 81.26 & 0 & 95.83 & 0 & 98.30 & 0 \\
\hline 1D-SSA & 85.50 & 13 & 95.53 & 7 & 98.52 & 13 \\
\hline F-1D-SSA & 85.78 & 12 & 95.55 & 7 & 98.50 & 12 \\
\hline 2D-SSA & 95.71 & 31 & 98.21 & 10 & 99.81 & 270 \\
\hline F-2D-SSA & 95.82 & 28 & 98.51 & 10 & 99.58 & 150 \\
\hline 2D-SSA-PCA & 97.61 & 31 & 99.58 & 10 & 99.83 & 270 \\
\hline F-2D-SSA-PCA & 97.59 & 28 & 99.59 & 10 & 99.88 & 150 \\
\hline 2D-EMD & 95.28 & 936 & 99.53 & 688 & 99.71 & 1506 \\
\hline
\end{tabular}

\section{Conclusions}

In HSI remote sensing, the 2D-SSA method has been proven really effective with relation to the current state of the art in extracting features from the hyperspectral cube. Nevertheless, its implementation requires band-based repetitions, since 2D-SSA has to be individually applied to every spectral scene in the cube. As this band-based implementation requires hundreds of individual SVDs, the computational complexity of the method can be remarkable under certain circumstances. In order to solve this drawback, a fast implementation F-2D-SSA is proposed in the present manuscript, where now a unique SVD analysis is required, leading to a single set of eigenvectors by which all spectral images in the cube are transformed. This particular SVD is applied to a representative scene from the cube, selected as the mean or the median scene out of the whole data set. Our experimental results show that F-2D-SSA is able to produce similar features with the same classification accuracy level in comparison to the conventional 2D-SSA (95.66\% and $95.82 \%$ instead of $95.71 \%)$, but with reduced computational complexity (saving factors of 10 and 100) and faster running time (reduced up to $60 \%$ ). 


\section{ACKNOWLEDGMENT}

This work was supported by the National Natural Science Foundation of China (61272381, 61571141), Guangdong Provincial Application-oriented Technical Research and Development Special Fund Project (2015B010131017), Science and Technology Major Project of Education Department of Guangdong Province (2014KZDXM060), the Natural Science Foundation of Guangdong Province (2016A030311013, 2015A030313672) and International Scientific and Technological Cooperation Projects of Education Department of Guangdong Province (2015KGJHZ021).

\section{REFERENCES}

[1] T. Qiao, J. Ren, C. Craigie, J. Zabalza, C. Maltin, and S. Marshall, "Singular spectrum analysis for improving hyperspectral imaging based beef eating quality evaluation," Computers and Electronics in Agriculture, 115: 21-25, 2015.

[2] T. Kelman, J. Ren, and S. Marshall, "Effective classification of Chinese tea samples in hyperspectral imaging," Artificial Intelligence Research, 2(4): 87-96, 2013.

[3] G. Lu, and B. Fei, "Medical hyperspectral imaging: a review," Journal of Biomedical Optics, 19(1), Jan. 2014.

[4] M. Sun, D. Zhang, Z. Wang, J. Ren, B. Chai, and J. Zhou, "What's Wrong with Murals at Mogao Grottoes: a Near-Infrared Hyperspectral Image Method," Scientific Reports, 5: Article number 14371, 2015.

[5] J. Ren, J. Zabalza, S. Marshall and J. Zheng, "Effective feature extraction and data reduction with hyperspectral imaging in remote sensing," IEEE Signal Processing Magazine, 31(4): 149-154, Jul. 2014

[6] L. Fang, S. Li, W. Duan, J. Ren, J. Atli Benediktsson, "Classification of hyperspectral images by exploiting spectral-spatial information of superpixel via multiple kernels," IEEE Trans. Geoscience and Remote Sensing, 53(12): 6663-6674, 2015

[7] N. Golyandina, A. Zhigljavsky, Singular spectrum analysis for time series, Springer, 2013.

[8] J. Zabalza, J. Ren, Z. Wang, S. Marshall, and J. Wang, "Singular spectrum analysis for effective feature extraction in hyperspectral imaging," IEEE Geoscience and Remote Sensing Letters, 11(11): 1886-1890, 2014.

[9] J. Zabalza, J. Ren, J. Han, H. Zhao, S. Li, and S. Marshall, "Novel two-dimensional singular spectrum analysis for effective feature extraction and data classification in hyperspectral imaging," IEEE Transactions on Geoscience and Remote Sensing, 53(8): 4418-4433, Aug. 2015.

[10] C. Rodarmel, and J. Shan, "Principal component analysis for hyperspectral image classification," Surveying and Land Information Science 62(2): 115-122, 2002 .

[11] B. Demir, and S. Ertürk, "Empirical mode decomposition of hyperspectral images for support vector machine classification," IEEE Transactions on Geoscience and Remote Sensing, 48(11): 4071-4084, 2010.

[12] R. D. Phillips, C. E. Blinn, L. T. Watson, and R. H. Wynne, “An adaptive noise-filtering algorithm for AVIRIS data with implications for classification accuracy," IEEE Transactions on Geoscience and Remote Sensing, 47(9): 3168-3179, Sep. 2009.

[13] M. Fauvel, J. A. Benediktsson, J. Chanussot, and J. R. Sveinsson, "Spectral and spatial classification of hyperspectral data using SVMs and morphological profile," IEEE Transactions on Geoscience and Remote Sensing, 46(11): 3804-3814, Nov. 2008. 
[14] J. Zabalza, J. Ren, Z. Wang, H. Zhao, J. Wang, and S. Marshall, "Fast implementation of singular spectrum analysis for effective feature extraction in hyperspectral imaging," IEEE Journal of Selected Topics in Earth Observation and Remote Sensing, vol. 8, no. 6, pp. 2845-2853, Jun. 2015.

[15] N. Golyandina, and K. D. Usevich, "2D-extension of singular spectrum analysis: algorithm and elements of theory," Matrix Methods: Theory, Algorithms, Applications World Scientific: 449-473. 2010.

[16] L. J. Rodriguez-Aragón, and A. Zhigljavsky, "Singular spectrum analysis for image processing,” Statistics and Its Interface, 3: 419-426. 2010.

[17] Pursue's university multispec site: June 12, 1992 aviris image Indian Pine Test Site [Online]. Available: https://engineering.purdue.edu/ biehl/MultiSpec/hyperspectral.html.

[18] Hyperspectral Remote Sensing Scenes [Online]. Available: http://www.ehu.es/ccwintco/index.php/Hyperspectral_Remote_Sensing_Scenes.

[19] R. O. Green et al, "Imaging spectroscopy and the airborne visible/infrared imaging spectrometer (AVIRIS)," Remote Sensing Environment 65:227-248 Elsevier Science Inc., 1998.

[20] R. Archibald and G. Fann. "Feature selection and classification of hyperspectral images with support vector machines." IEEE Geoscience and Remote Sensing Letters, 4(4), Oct. 2007.

[21] S. Holzwarth et al. "HySens - DAIS 7915/ ROSIS Imaging Spectrometers at DLR," Presented at the 3rd EARSeL Workshop on Imaging Spectroscopy, Herrsching, 13-16 May 2003.

[22] G. Mountrakis, J. Im, and C. Ogole, "Support vector machines in remote sensing: a review," ISPRS Journal of Photogrammetry and Remote Sensing, $66: 247-259,2011$.

[23] J. Zabalza, J. Ren, C. Clemente, G. Di Caterina, and J.J. Soraghan, "Embedded SVM on TMS320C6713 for signal prediction in classification and regression applications," in 5th European DSP Education and Research Conf., Amsterdam, Sep. 2012.

[24] J. Zabalza, C. Clemente, G. Di Caterina, J. Ren, J. J. Soraghan, and S. Marshal, "Robust PCA micro-Doppler classification using SVM on embedded systems," IEEE Transactions on Aerospace and Electronic Systems, 50(3), Jul. 2014.

[25] Chih-Chung Chang and Chih-Jen Lin, LIBSVM: a library for support vector machines. ACM Transactions on Intelligent Systems and Technology, 2(3): 27 pages, 2011. Software available at http://www.csie.ntu.edu.tw/ cjlin/libsvm.

[26] G. M. Foody, "Thematic map comparison: Evaluating the statistical significance of differences in classification accuracy," Photogramm. Eng. Remote Sens., 70(5): 627-633, May 2004.

[27] A. Korobeynikov, “Computation-and space-efficient implementation of SSA," arXiv preprint arXiv: 0911.4498, 2009.

[28] G. Golub, and C. Reinsch, "Singular value decomposition and least squares solutions," Numer. Math. 14:403-420, 1970. 